\title{
Metástasis cervical con primario desconocido, cambios en los paradigmas. Revisión sobre el enfrentamiento clínico, estudio y tratamiento
}

\section{Cervical metastases from unknown primary tumor, paradigm changes. Review on clinical confrontation, study and treatment}

\author{
Paul Boettiger B. ${ }^{1}$, Ignacio Rey D. ${ }^{1}$, Cristofer Salazar F. ${ }^{1}$, \\ Mariana Marqués H. ${ }^{2}$, Ricardo Zamorano ${ }^{1}{ }^{1}$
}

\section{Resumen}

En adultos, una masa cervical detectada mediante examen físico o un estudio de imagen puede ser la única manifestación de un cáncer proveniente de cabeza y cuello. Un retraso en el diagnóstico repercute en el pronóstico de la enfermedad, por lo que debe haber un alto índice de sospecha. Las metástasis cervicales con primario desconocido (MC$\mathrm{CPD}$ ) son tumores metastásicos en los que el estudio diagnóstico no logró identificar el sitio primario del cáncer, con una histología predominantemente de tipo escamosa. Según algunos estudios, el origen más frecuente resultó ser la orofaringe, incluyendo amígdala palatina y base de lengua. Factores de riesgo conocidos son edades avanzadas, consumo de tabaco y de alcohol. Actualmente, la infección por el virus del papiloma humano (VPH) está teniendo un rol cada vez más importante como factor de riesgo, formando parte de entre 20\%-25\% de los cánceres de cabeza y cuello. Al enfrentarse a un paciente con masa cervical es importante realizar una completa anamnesis y examen físico acucioso para detectar cualquier elemento sugerente de malignidad. Se debe complementar con nasofibroscopía para visualizar estructuras que no alcanzan a evaluarse en el examen habitual. También se puede orientar la búsqueda del primario desconocido en base a los patrones de drenaje linfático. Dentro del estudio complementario se puede comenzar con una tomografía computada (TC) y se puede considerar también el ultrasonido o un PET/TC. Si con esto aún no se logra definir el primario, continuar con una punción aspirativa con aguja fina (PAAF), luego biopsia core que consiste en tomar una muestra del centro de la lesión guiada por ecografía, si fuese necesario, incluyendo inmunohistoquímica para VPH; ambos estudios histológicos son preferibles en vez de una biopsia abierta debido al menor riesgo de diseminación y complicaciones. El siguiente paso incluye estudio endoscópico y biopsias bajo anestesia. El tratamiento de los pacientes con MCCPD, va a depender de factores relacionados con el estadio de la enfermedad: desde cirugía o radioterapia (RT) únicas, cirugía más RT, y en algunos casos quimioterapia. Se recomienda seguimiento clínico frecuente durante los primeros años y con imágenes dentro de los 6 primeros meses postratamiento.

Palabras clave: masa en el cuello, carcinoma de células escamosas, carcinoma de origen primario desconocido, cabeza y cuello, virus del papiloma humano.

\begin{abstract}
In adults, a cervical mass detected by physical examination or an imaging study may be the only manifestation of cancer from the head and neck. A delay in the diagnosis affects the prognosis of the disease, so there must be a high index of suspicion. Cervical metastases from unknown primary tumor (CUP) are metastatic tumors in which the diagnostic study failed to identify the primary site of cancer, with predominantly squamous histology. According to some studies, the most frequent origin was the oropharynx, including palatine tonsil and tongue base. Known risk factors are advanced ages, tobacco and alcohol consumption. Currently, human papilloma virus (HPV) infection is playing an increasingly important role
\end{abstract}

\section{${ }^{1}$ Departamento de} Otorrinolaringología, Hospital Clínico Universidad de Chile. Santiago, Chile.

${ }^{2}$ Escuela de Medicina, Facultad de Medicina, Universidad de Chile. Santiago, Chile.

Los autores declaran no tener conflicto de interés.

Recibido el 14 de noviembre de 2019. Aceptado el 4 de mayo de 2020 .

Correspondencia: Paul Boettiger B Santos Dumont 999, Hospital Clínico Universidad de Chile. Santiago, Chile. Email: paulboettiger@gmail. com 
as a risk factor, being the cause of between 20-25\% of cancers of the head and neck. When confronting a patient with cervical mass it is important to carry out a complete anamnesis and a thorough physical examination to detect any element suggestive of malignancy. Physical examination could be complemented with a flexible nasal endoscopic to evaluate structures that can not be evaluated in the habitual examination. The search for the unknown primary can also be oriented based on lymphatic drainage patterns. Within the complementary evaluations, one can start with a study of images such as computed tomography (CT) or magnetic resonance imaging (MRI) with contrast, and also could consider ultrasound or PET/CT. If the primary can not be defined yet, fine needle aspiration (FNAP) can be the next choice and then a core biopsy that consisting of taking a sample from the center of the ultrasound-guided lesion, if necessary, including immunohistochemistry for $H P V$; both histological studies are preferable to an open biopsy because of the lower risk of complications. The next step searching for the primary includes endoscopic study and biopsies under anesthesia. Regarding to the management of patients with CUP, it will depend on factors related to the stage of the disease: from surgery or radiotherapy $(R T)$ only, surgery and RT, and in some cases chemotherapy. Frequent clinical follow-up is recommended during the first years and images within the first 6 months after treatment.

Keywords: neck mass, squamous cell carcinoma, carcinoma of unknown primary, head and neck, human papillomavirus.

\section{Introducción}

Una masa de cuello se puede definir como una lesión anormal (congénita o adquirida) que es visible, palpable o detectada en un estudio de imágenes ${ }^{1}$. En niños la principal causa de masa cervical persistente es una patología infecciosa, mientras que, en adultos, una masa cervical asintomática puede ser la manifestación inicial o la única manifestación de un cáncer de cabeza y cuello ${ }^{1}$. Es por esto que en adultos el índice de sospecha de una neoplasia oculta tiene que ser alto, para poder hacer un diagnóstico precoz, ya que el retraso afecta directamente al paciente empeorando su pronóstico ${ }^{1}$. Se estima que hace 40 años, el retraso en el diagnóstico de malignidad de una masa del cuello en promedio era de 5-6 meses desde el momento de la presentación inicial, hoy en día los estudios publicados continúan informando un retraso entre 3-6 meses $^{1}$. Dentro de los diagnósticos diferenciales de una masa cervical encontramos la metástasis cervical con primario desconocido (MCCPD), definida como un grupo heterogéneo de tumores metastásicos para los cuales un estudio diagnóstico estandarizado no identifica el sitio primario ${ }^{2}$. Esta entidad tiene una incidencia de 3\%-9\% de todos los cánceres de cabeza y cuello y la histología escamosa constituye el $75 \%$ de los casos ${ }^{3}$. Issing y cols. analizaron 167 pacientes con MCCPD, el origen más frecuente del primario fue la orofaringe $(38,9 \%)$, incluida la amígdala palatina y la base de la lengua, seguida de hipofaringe $(22,3 \%)$, nasofaringe $(11,1 \%)$ y laringe $(11,1 \%)$. Cianchetti y cols. evaluaron a 236 pacientes, encontrando que el origen más frecuente eran la fosa amigdalina $(44,7 \%)$ y la base de la lengua $(43,9 \%)^{2}$.

Antiguamente los factores de riesgo para una MCCPD eran ser hombre entre 55 y 65 años, con historia de tabaquismo y alcohol ${ }^{4}$. Hoy en día y debido a la influencia de los cánceres relacionados con virus papiloma humano $(\mathrm{VPH})$, los pacientes son más jóvenes, con frecuencia carecen de exposición a tabaco y alcohol, hay mayor consumo de marihuana, más parejas sexuales (orales y vaginales), mejor dentición, mayor nivel de educación y estatus socioeconómico ${ }^{1}$. Con respecto al VPH, es importante señalar que la incidencia de cáncer orofaríngeo $\mathrm{VPH}(+)$ ha aumentado más del doble, mientras que los VPH (-) han disminuido a la mitad ${ }^{1}$. Se ha encontrado que el VPH-16 causa entre un 20 a 25\% de los carcinomas de células escamosas de cabeza-cuello, y un $40 \%$ a $60 \%$ de los de orofaringe 5 . En los últimos años el advenimiento de nuevos conocimientos en cáncer de cabeza y cuello, como la influencia del VPH y el virus de Epstein-Barr (VEB), o nuevas técnicas como la cirugía robótica transoral (TORS) o cirugía láser transoral (TOLS), están haciendo que los paradigmas establecidos en el manejo de la MCCPD hoy en día tengan que ser replanteados. 


\section{Enfrentando un paciente con masa cervical}

\section{Historia y examen físico}

Debe ser riguroso para identificar a aquellos con mayor riesgo de malignidad. Si bien la clínica es una prueba imperfecta para identificar malignidad en un ganglio cervical, el bajo costo y la valiosa información que podemos obtener de éste, transforman al examen físico en la primera herramienta que nos puede permitir acelerar el diagnóstico específico de una masa cervical (Tabla 1) $)^{1,6}$.

\section{Uso de antibióticos}

En adultos, no existe evidencia de uso de antibióticos como enfrentamiento inicial ante una masa cervical, por lo tanto, no se deben recetar de forma rutinaria, a menos que haya signos y síntomas que sugieran

Tabla 1. Hallazgos sugerentes de malignidad en el estudio de una masa cervical ${ }^{1}$

\section{Características de la masa cervical}

Masa presente por $\geq 2$ semanas o de Una masa persistente tiene mayor probabilidad de ser maligna temporalidad incierta

Masa poco móvil Las metástasis pueden infiltrar la cápsula del linfonodo e invadir directamente las estructuras adyacentes lo que la hace ser poco móvil

Masa de consistencia firme Un linfonodo maligno suele ser firme debido a la ausencia de edema tisular, por el contrario, una adenopatía infecciosa suele ser blanda debido al edema tisular

Masa $>1,5 \mathrm{~cm}$

Piel ulcerada sobre la masa $\quad$ Puede ser debido a una metástasis cervical que puede atravesar la cápsula del linfonodo e invadir y necrosar directamente la piel. Otra causa es que la ulceración secundaria a un cáncer de piel tenga una extensión directa al cuello

Historia y/o examen físico sugerentes de malignidad en el contexto de una masa cervical

Edad > 40 años

Consumo de tabaco y alcohol

Otalgia ipsilateral a la masa

Lesiones en cavidad oral $u$ orofaríngea

Disfagia

Cambios en la voz, dolor al fonar

Hipoacusia ipsilateral a la masa de inicio reciente

Obstrucción nasal y/o epistaxis ipsilateral a la masa

Pérdida de peso inexplicable

Antecedente de cáncer de cabeza y cuello previo

Masa indolora

Lesiones cutáneas en cabeza y cuello
Edades avanzadas se asocian con un mayor riesgo de cáncer escamoso de cabeza y cuello

Tabaco y alcohol son factores de riesgo sinérgicos para cáncer escamoso de cabeza y cuello

Otalgia con otoscopía normal podría corresponder a una otalgia referida proveniente de la faringe, sobre todo en particular a la hipofaringe. El hallazgo de otitis media con efusión podría deberse a disfunción de la tuba auditiva por obstrucción secundario a cáncer de nasofaringe

Úlcera o masa, sensible a la palpación, o disminución de la movilidad de la lengua podrían indicar malignidad

Podría ser causada por una lesión ulcerada o tumoral, o alguna disfunción del sistema aerodigestivo

Podría corresponder a cáncer proveniente de la laringe o estructuras adyacentes

Podría corresponder a cáncer proveniente de la nasofaringe asociado a una otitis media con efusión

Podría corresponder a cáncer proveniente de la nariz o nasofaringe

Un cáncer independiente de su localización puede producir una pérdida de peso importante, pero en cabeza y cuello particularmente puede causar dificultad para deglutir y con ello favorecer la pérdida de peso debido a una disminución del aporte dietético

Un cáncer previo de cabeza y cuello implica riesgo de recurrencia local o regional (ganglionar) o desarrollar un segundo primario. Pacientes con radioterapia previa también tienen riesgo de una neoplasia secundaria años después

Una masa inflamatoria o infecciosa suele ser dolorosa o sensible. Una masa no sensible a la palpación es más probable de ser neoplásica

El cáncer de piel puede dar metástasis a linfonodos cervicales 
infección bacteriana. El uso prudente de los antibióticos minimiza el riesgo de presentar efectos secundarios, minimiza el riesgo de desarrollar resistencia bacteriana, disminuye los costos innecesarios relacionados a la atención de salud y disminuye el diagnóstico tardío de enfermedades malignas ${ }^{1}$.

\section{Enfrentamiento inicial frente a una masa cervical sospechosa}

Historia clínica y examen físico acucioso incluida una nasofibroscopía. Esta nos permitirá evaluar de forma detallada desde fosas nasales hasta la laringe, haciendo énfasis en el septum, piso nasal, cornetes, tuba auditiva, nasofaringe, senos piriformes, pared posterior de la hipofaringe, epiglotis, cuerdas vocales y subglotis ${ }^{1}$. Una forma de buscar el primario desconocido de la masa cervical es conociendo los patrones de drenaje linfático habituales (Tabla 2), ya que estos en general tienen un patrón constante $e^{1,3,7}$.

\section{Rol de las imágenes en MCCPD}

Nos permitirán localizar y caracterizar la masa cervical sospechosa, evaluar otras masas no palpables y evaluar los potenciales sitios primarios ${ }^{1,4}$. Solicitar tomografía computada (TC) o resonancia magnética (RM) de cuello con contraste como primer estudio complementario ${ }^{1,3,8}$, se deben realizar antes de cualquier procedimiento invasivo para evitar los falsos positivos $\mathrm{u}$ otras interpretaciones erróneas por inflamación secundaria a la biopsia $^{4}$. Otro aspecto relevante del estudio de imágenes es caracterizar la arquitectura nodal y la presencia y/o extensión de la diseminación tumoral extracapsular, ya que el compromiso de estructuras críticas, como la arteria carótida, influirá en la decisión de realizar o no una disección del cuello ${ }^{3,4,8}$.

Otros estudios de imágenes que podrían ser considerados son la TC de tórax en el contexto de un paciente con riesgo de padecer cáncer pulmonar primario. El ultrasonido nos permitirá caracterizar la masa cervical, buscar masas similares no detectadas, será la primera opción para evaluar masas tiroideas y glándulas salivales y serviría como guía en la punción con aguja fina ${ }^{1,4}$. El PET/TC, ante una histología de cáncer escamoso, su gran utilidad estaría centrada en dirigir el mapeo biópsico, pero no reemplazarlo, mientras que en otras histologías aporta menos, por ejemplo, en sarcomas o glándulas salivales ${ }^{6,9}$. Con respecto a este y teniendo en cuenta su alta tasa de falsos positivos y negativos ${ }^{4,7}$, el PET/ TC podría tener alguna utilidad en encontrar

Tabla 2. Patrones comunes de linfonodos metastásicos en cabeza y cuello ${ }^{4,7}$

\begin{tabular}{|c|c|}
\hline Grupo linfonodal & Sitios de tumor primario \\
\hline Nivel IA (submentoniano) & Cavidad oral anterior (incisivos, caninos), labio inferior, mentón, punta nasal \\
\hline Nivel IB (submandibular) & $\begin{array}{l}\text { Cavidad oral (premolar, molares), cavidad nasal anterior, glándulas } \\
\text { submandibulares, piel de la zona media facial (mejillas), porción anterior de la } \\
\text { lengua }\end{array}$ \\
\hline $\begin{array}{l}\text { Nivel II (yugular superior) } \\
\text { - IIA (anterior al N. Espinal accesorio) } \\
\text { - IIB (posterior al N. Espinal accesorio) }\end{array}$ & $\begin{array}{l}\text { Orofaringe, cavidad oral, nasofaringe, cavidad nasal, laringe, hipofaringe, base } \\
\text { de lengua } \\
\text { Orofaringe, porción anterior y posterior de la lengua } \\
\text { Orofaringe, parótida }\end{array}$ \\
\hline Nivel III (yugular medio) & Orofaringe, cavidad oral, nasofaringe, laringe, hipofaringe \\
\hline Nivel IV (yugular inferior) & Orofaringe, laringe, hipofaringe \\
\hline Nivel V (triángulo posterior) & Nasofaringe, cuero cabelludo posterior, tiroides \\
\hline Nivel VI (compartimento anterior-central) & Tiroides, laringe, hipofaringe \\
\hline Supraclavicular & Tiroides, metástasis originadas en órganos bajo la clavícula (tórax, abdomen, etc.) \\
\hline Retrofaríngeo & Nasofaringe, faringe posterior \\
\hline Parotídeo & Piel y cuero cabelludo de la zona superolateral de la cabeza, parótidas \\
\hline
\end{tabular}


el primario porque puede ayudar a identificar lesiones pequeñas mayores a $1 \mathrm{~cm}^{3}$; y en pacientes con alto riesgo de metástasis, como es el caso de los carcinomas de hipofaringe y nasofaringe, permitiría evaluar los posibles primarios. Aún así hay un porcentaje de tumores que no serán identificados a pesar de tener un PET/TC ${ }^{1,4,6,8,9}$.

\section{TC o RM ¿cuál elegir?}

La TC tiene varios beneficios que respaldan su uso como primera opción ${ }^{1}$. Dentro de las ventajas de la TC, destaca ser un examen con mayor disponibilidad, menos costoso, mejor tolerado por los pacientes debido al menor tiempo de escaneo y mayor espacio físico dentro de la máquina de escáner; sus desventajas es que utiliza radiación ionizante con dosis promedio de $3 \mathrm{mSv}$ (equivalente a 150 radiografías) $^{1}$. Las ventajas de la RM es que tiene un mejor contraste tisular, por lo que puede ayudar a detectar tumores subclínicos que no son evidentes en la endoscopia nasal. Es más útil cuando se sospecha tumores primarios de nasofaringe, compromiso de nervios craneanos o diseminación perineural. Sus desventajas es que es un examen que toma más tiempo, tiene artefactos por los movimientos, no se puede aplicar en pacientes con incapacidad para mantenerse inmóviles o claustrofóbicos y está contraindicado en pacientes que tengan algún dispositivo implantado ${ }^{1,4}$. Independiente si se realiza una TC o una RM, siempre se debe usar contraste endovenoso a menos que haya una contraindicación. El contraste mejora las características de la masa, distingue quistes de lesiones sólidas, ayuda a definir los bordes de la lesión y mejora la identificación de los sitios primarios pequeños ${ }^{1}$.

\section{Qué hacer si todo lo anterior no es suficiente para definir la etiología de la masa cervical}

La punción aspirativa con aguja fina (PAAF) es el siguiente paso para identificar la naturaleza de la lesión cervical, ya que nos ayuda a saber la histología, sobre todo si nos vemos enfrentados a un cáncer escamoso. Con la PAAF no se realiza una apertura de la cápsula, por lo que no se compromete la sobrevida del paciente en comparación a una biopsia abierta ${ }^{1,4,6}$.

\section{Rol de la PAAF en MCCPD}

En manos de médicos familiarizados con esta técnica, la PAAF se puede realizar en la primera consulta, incluso antes de solicitar imágenes adicionales. Es un procedimiento seguro, con bajo riesgo de siembra tumoral $(0,00012 \%)^{1}$, no hay contraindicaciones absolutas para este procedimiento, incluso en pacientes anticoagulados. En caso de sospechar una lesión vascular, se sugiere realizar imágenes antes de la punción. Para mejorar el rendimiento de este procedimiento, se recomienda realizarla guiada por ultrasonido, sobre todo en lesiones quísticas o necróticas ${ }^{1}$. La sensibilidad global de este procedimiento es de $89,6 \%$; especificidad $96,5 \%$; valor predictivo positivo $96,2 \%$; y valor predictivo negativo $90,3 \%$. Con histopatólogos experimentados, este examen alcanza una sensibilidad diagnóstica de $83 \%$ a $97 \%$ y una especificidad de $91 \%$ a $100 \%$ para lesiones metastásicas ${ }^{1,4,6}$.

\section{Exámenes complementarios a la PAAF}

El diagnóstico preciso dependerá de la capacidad para realizar pruebas complementarias a nuestra muestra. Si bien hay varias pruebas complementarias a la PAAF que pueden ayudar en el diagnóstico (Tabla 3), como mínimo una parte de la muestra debe enviarse en formalina u otro fijador adecuado para realizar una hibridación in situ/PCR del VPH y/o la inmunohistoquímica de p16. También se sugiere estudiar con hibridación in situ del virus de Epstein-Barr según la clínica del paciente ${ }^{1,6}$. Estos análisis en el contexto de una MCCPD podría ayudar a identificar la ubicación del tumor primario (orofaringe versus otros y desconocidos) y predecir los resultados de supervivencia del paciente ${ }^{5}$.

\section{Cuándo repetir la PAAF y otras alternativas}

Cuando la PAAF no es suficiente, los resultados son inadecuados, la citología es indeterminada o arroja un diagnóstico de patología benigna, se recomienda la repetición de ésta por sobre una biopsia abierta, ya que el repetirla aumenta la probabilidad de pesquisar una neoplasia maligna ${ }^{1,4}$. La biopsia core es una opción después de una PAAF con resultado inadecuada o indeterminada. En un metaanálisis, se demostró que la biopsia core guiada por ultrasonido tiene una alta tasa 
de precisión (94\% y 96\% en la detección de neoplasia y malignidad, respectivamente), así como una tasa baja de complicaciones $(1 \%)^{1}$. Si la historia clínica y los hallazgos en el examen físico sugieren un linfoma, entonces una biopsia core se puede considerar como la técnica de primera línea. En este contexto, la biopsia core tiene una sensibilidad más alta que la PAAF $(92 \% \text { frente a } 74 \%)^{1}$.

\section{Si no logramos hacer un diagnóstico con examen físico, TAC, PET/CT, PAAF, corresponde realizar un mapeo con biopsias al azar}

Realizar una exploración endoscópica exhaustiva bajo anestesia, para visualizar nariz, nasofaringe, orofaringe, hipofaringe, laringe y eventuales biopsias, de cualquier área sospechosa $^{3}$. Este procedimiento consiste en realizar palpación de las amígdalas, base de la lengua y la pared posterior de la faringe, laringoscopia directa, nasofibroscopía, una esofagoscopia especialmente en los casos en que tengamos un adenopatía supraclavicular en el grupo IV, y una broncoscopía cuando tengamos hallazgos sospechosos en las imágenes de tórax ${ }^{1,3,4,6,10}$. Se busca encontrar el primario y así evitar realizar una biopsia quirúrgica de cuello ${ }^{1,4}$. De encontrar una lesión sospechosa, se tiene que tomar una biopsia de esta y estudiarla mediante biopsia rápida ${ }^{1,3,7}$. En caso contrario, se tiene que tomar biopsias aleatorias de los sitios primarios más probables ${ }^{4}$. Más del $80 \%$ de los tumores primarios, cuando se encuentran, residirán en las amígdalas y en la base de la lengua. Más recientemente, varios autores han demostrado que las amígdalas palatinas pueden albergar el tumor primario oculto en hasta el 30\%-40\% de los casos, y en el 10\%-25\% de los casos el carcinoma primario se puede localizar en la amígdala contralateral a la masa del cuello. Esto ha llevado a la recomendación de realizar una amigdalectomía bilateral junto con la panendoscopía, biopsia de la base lengua y nasofaringe ${ }^{10}$.

Si tenemos compromiso de los niveles ganglionares II o III, se sugiere hacer una amigdalectomia bilateral asociada a una mucosectomía de base lengua ipsilateral a la masa, ya que se sufre riesgo de estenosis faríngea cuando va asociado a amigdalectomía bilateral ${ }^{4,7}$. Se sugieren hacer mediante cirugía robótica transoral (TORS) o cirugía láser transoral
(TOLS), dado que se asocia a una tasa mucho más alta de detección de tumores primarios en comparación a la evaluación endoscópica tradicional ${ }^{3,7}$.

La imagen de banda estrecha (NBI) es una herramienta que permitiría mejorar el rendimiento en la evaluación endoscópica. En un estudio se demostró que utilizando la imagen de banda estrecha todas las lesiones malignas mostraron un patrón de proliferación microvascular ${ }^{4,11}$ la que los haría más evidente al momento de la evaluación. La biopsia a ciegas de la nasofaringe, el seno piriforme y la hipofaringe, en ausencia de hallazgos clínicos o radiográficos sospechosos, ya no están indicadas debido a la baja incidencia de cánceres en estos subsitios ${ }^{6}$.

Biopsia abierta es la última alternativa, podría realizarse en el mismo examen bajo anestesia, con el consentimiento y participación del paciente en el proceso de decisión. Para la mayoría de las masas, la incisión debe planearse de modo que pueda extenderse a una incisión más grande si alguna vez se realiza una disección del cuello. Debe realizarse con biopsia rápida y si el resultado es un cáncer escamoso, se procede a realizar vaciamiento funcional. La biopsia por escisión es preferible para prevenir el derrame tumoral, especialmente con respecto a las masas quísticas, pero puede no ser factible en casos de masas grandes, sólidas o adheridas a estructuras vitales donde aumenta el riesgo de sangrado y/o lesión nerviosa ${ }^{1}$.

\section{Tratamiento del paciente con MCCPD}

El manejo de los pacientes con MCCPD es un gran desafío en oncología. Las recomendaciones incluyen cirugía sola, radioterapia de campo limitado e irradiación profiláctica extensa de todos los sitios potenciales de mucosa, así como ambos lados del cuello con o sin quimioterapia concomitante. Sin embargo, la morbilidad de estos tratamientos combinados puede ser alta, disminuyendo la calidad de vida de los pacientes, sobre todo si son relativamente jóvenes. No hay estudios aleatorizados o prospectivos disponibles para respaldar ninguno de estos enfoques específicamente ${ }^{3,12}$. Al momento de definir el tratamiento para nuestros pacientes, es necesario tener en cuenta los principales factores pronósticos en la 
MCCPD. Dentro de los factores significativos para tener una mejor sobrevida global y una supervivencia sin recurrencias son la ausencia de diseminación extracapsular, el estado de resección ( $\mathrm{R} 0$ versus $\mathrm{R} 1 / \mathrm{R} 2$ ), el nivel de ganglios linfáticos comprometidos en el cuello (I-III versus $\mathrm{IV}-\mathrm{V}$ ) y una mayor puntuación en el índice de Karnofsky ${ }^{2,6,11}$.

Factores significativos para tener una peor sobrevida global y supervivencia libre de recurrencia son el presentar resección R1/ R2 y compromiso de grupo IV de ganglios linfáticos ${ }^{2}$. Hay que tener en consideración que una biopsia escisional en la MCCPD es controvertida debido a la alta incidencia de recidiva local. Sin embargo, se indica si existe una alta sospecha de linfoma, sarcoma, melanoma o adenocarcinoma. Los pacientes deben estar preparados para la disección del cuello en el momento de la biopsia abierta ${ }^{3}$.

A continuación, se describen las principales recomendaciones de tratamiento según el estadio de la enfermedad:

\section{Tratamiento de modalidad única}

Enfermedad del cuello poco avanzada, como los casos de N1 y N2a sin extensión extracapsular (ECE) en el examen histopatológico, muchos estudios informaron un excelente control regional tanto con cirugía exclusiva como con RT exclusiva ${ }^{4,11,13}$. Una posible ventaja de la RT sobre la cirugía es que permite el tratamiento de sitios de la mucosa en riesgo con una dosis subclínica de radiación ${ }^{4}$.

Las ventajas con la cirugía radican en que nos permite realizar una etapificación de la enfermedad nodal de forma más precisa. Davidson y cols. encontró que el $34 \%$ de los pacientes con MCCPD que se clasificaron inicialmente como N1 o N2a tenían múltiples nodos en la disección del lado ipsilateral del cuello. Además, la cirugía puede ayudar a decidir la indicación de la RT postoperatoria porque puede proporcionar el estadio $\mathrm{pN}$, el grado histológico, la extensión extracapsular, la invasión linfovascular y la invasión perineural. Por último, cabe destacar que una disección selectiva del cuello tiene una tasa muy baja de complicaciones postoperatorias, es menos costosa que la RT y puede eliminar la carga tumoral gruesa y las micrometástasis del cuello $\mathrm{o}^{4,6,13,14}$.

\section{Tratamiento con modalidad combinada}

Enfermedad de cuello avanzada o con el antecedente de una biopsia incisional o escisional de cuello, la terapia de modalidad combinada parece ser lo más indicado, pero no hay certeza sobre cuál es el mejor momento para realizar la cirugía y la $\mathrm{RT}^{4,11,13,15}$. Al momento del diagnóstico de MCCPD, la etapa $\mathrm{N}$ en la mayoría de los casos está N2a, N2b y N2c, con un tamaño medio de ganglios linfáticos de 3,5-5 cm. Por lo tanto, la mayoría de los pacientes con MC$\mathrm{CPD}$ reciben tratamiento combinado en forma de resección seguida de radiación adyuvante (quimioterapia) o quimiorradiación primaria (disección cervical posterior a la terapia). En términos de resultados y según la evidencia existente, no está claro cuál representa la mejor opción y la decisión se toma caso a caso en comité oncológico ${ }^{4,11}$.

\section{Radioterapia unilateral versus bilateral}

Otra discusión es que los candidatos para RT bilateral de cuello son pacientes con sospecha de tumores primarios localizados cerca de la línea media (es decir, base de la lengua en casos de VPH positivos o después de una amigdalectomía que no logró identificar el primario; tumor de nasofaringe en casos VEB positivos, y/o estudios de imagen sugerentes de lesión, pero con biopsia negativa) o aquellos con enfermedad nodal extensa que hace que el cuello contralateral tenga un riesgo mayor de metástasis ganglionares ocultas, o pacientes con ganglios bilaterales ${ }^{4,13}$. En el caso de un paciente joven con buena salud general, debe considerarse una irradiación completa, en contraste, se puede recomendar la irradiación ipsilateral exclusiva para pacientes con índice de Karnofsky muy bajo o muy longevos ${ }^{3}$. Idealmente se debe preferir la radioterapia de intensidad modulada (IMRT), la superioridad de esta, es la cobertura del territorio objetivo y la preservación de los órganos en riesgo, esto puede ser beneficioso para los pacientes con MCCPD.

\section{Irradiamos o no la mucosa}

La tendencia actual es a limitar el campo de radiación. La elección de un enfoque menos agresivo se basa en el compromiso o extensión de la enfermedad del cuello. MCCPD limitadas al grupo II y en menor medida nivel III, tiene 
indicación de irradiar mucosa orofaríngea bilateral, a pesar de la baja incidencia de cáncer de amígdala. Irradiar nasofaringe es poco común y algunos profesionales omiten esta área del campo de tratamiento en pacientes seleccionados, particularmente aquellos con enfermedad ganglionar $\mathrm{p} 16+^{4}$.

\section{El rol de la quimioterapia}

Los casos en donde con mayor frecuencia se ha visto el uso de quimioterapia es en la extensión extracapsular y en la enfermedad residual después de la cirugía de cuello. Otros casos es la presencia de metástasis nodales no resecables o de alto volumen (N2-N3), donde la eficacia de la quimiorradiación como terapia inicial también se ha demostrado ${ }^{3,4,6,13,14}$. La elección de la quimioterapia debe ser individualizada en función de las características del paciente, estado funcional, la histología, y también de los objetivos que se busca con la terapia ${ }^{3}$.

\section{Seguimiento}

Se sugiere cada 1-3 meses durante el primer año, cada 2-4 meses durante el segundo año, cada 4-6 meses hasta los cinco años y cada 6-12 meses a partir de entonces. El control con imágenes posterior al tratamiento es con TC o RM, se recomienda dentro de los 6 meses posteriores al tratamiento ${ }^{3}$. Se describe en la literatura que el PET-TC tiene una mejor sensibilidad y especificidad que la TC o RM para el control a las 12 semanas. Si PET-TC es negativo el valor predictivo negativo es casi $100 \%{ }^{9}$.

\section{Conclusiones}

El MCCPD es una condición poco frecuente con una incidencia que va en aumento, y de la cual no existe evidencia definitiva tanto para el diagnóstico como para el tratamiento ${ }^{4}$.

$\mathrm{Al}$ momento de enfrentarnos a una MC$\mathrm{CPD}$, los diagnósticos diferenciales deben incluir tanto lesiones benignas como malignas ${ }^{7}$. En pacientes adultos, la mayoría de los casos será un carcinoma células escamoso VPH +, por lo que el principal sitio a evaluar será las amígdalas palatinas y la base de la lengua. Un avance en el estudio de la MCCPD es la inmunohistoquímica para VPH a través del p16, ya que al identificar este marcador en los ganglios metastásicos nos ayudaría a localizar los sitios de los tumores primarios y a predecir el pronóstico del paciente ${ }^{5,7}$.

Se debería ofrecer sistemáticamente un diagnóstico exhaustivo y preciso. El estudio debería incluir una historia y examen físico detallado, nasofibroscopía, TC o RNM, PAAF con inmunohistoquímica, PET TC y una evaluación endoscópica bajo anestesia con eventual toma de biopsias de lesiones sospechosas o en caso de no tener lesiones sospechosas, realizar una amigdalectomía al menos ipsilateral más resección de la base de la lengua a través del TORS o TOLS.

Los pacientes con enfermedad de cuello con bajo volumen, estadios N1 y N2a y sin extensión extracapsular en el examen histopatológico deben recibir tratamiento de modalidad única. La radioterapia y la cirugía pueden ser igualmente efectivas, pero si es posible, se debe favorecer la cirugía porque nos entrega una estadificación más precisa ${ }^{4}$. Los pacientes con condiciones más avanzadas requieren tratamiento combinado en la forma de resección seguida de radiación adyuvante o quimiorradiación primaria (disección de cuello posterior a la terapia $)^{4}$.

\section{Bibliografía}

1. Pynnonen MA, Gillespie MB, Roman B, et al. Clinical Practice Guideline: Evaluation of the Neck Mass in Adults. Otolaryngol Neck Surg 2017;157:S1-S30.

2. Kaizu H, Ogino I, Hata M, et al. Chemoradiation as a definitive treatment for cervical lymph node metastases from unknown primary cancer. Anticancer Res 2013;33:5187-5192.

3. Cerezo L, Raboso E, Ballesteros AI. Unknown primary cancer of the head and neck: a multidisciplinary approach. Clin Transl Oncol 2011;13:88-97.

4. Arosio AD, Pignataro L, Gaini RM, Garavello W. Neck lymph node metastases from unknown primary. Cancer Treat Rev 2017;53:1-9.

5. Park GC, Lee M, Roh J-L, et al. Human papillomavirus and p16 detection in cervical lymph node metastases from an unknown primary tumor. Oral Oncol 2012;48:1250-1256.

6. Eskander A, Ghanem T, Agrawal A. AHNS Series: Do you know your guidelines? Guideline recommendations for head and neck cancer of unknown primary site. Head Neck 2018; 40:614-621.

7. Chernock RD, Lewis JS. Approach to Metastatic Carcinoma of Unknown Primary in the Head and 
Neck: Squamous Cell Carcinoma and Beyond. Head Neck Pathol 2015;9:6-15.

8. Hermans R. Imaging in cervical nodal metastases of unknown primary. Cancer Imaging 2011;11A:S9-14.

9. Boettiger BP, Zamorano SR, Miranda G. PET-TC, rol y fundamentos de su uso en la especialidad de otorrinolaringología y cirugía de cabeza y cuello. Rev Otorrinolaringol Cir Cabeza Cuello 2018;78:221-227.

10. Mohindra S, Bhattacharya A, Goshal S, Gupta B. Incremental (?) role of positron emission tomography/computed tomography in clinically unknown primary patients with neck metastasis. Indian J Cancer 2014;51:142-144.

11. Yamazaki T, Kodaira T, Ota Y, et al. Retrospective analysis of definitive radiotherapy for neck node metastasis from unknown primary tumor: Japanese Radiation Oncology Study Group study. Jpn J Clin Oncol 2017;47:856-862.
12. Demiroz C, Vainshtein JM, Koukourakis GV, et al. Head and neck squamous cell carcinoma of unknown primary: Neck dissection and radiotherapy or definitive radiotherapy. Head Neck 2014; 36:15891595.

13. Strojan P, Ferlito A, Langendijk JA, et al. Contemporary management of lymph node metastases from an unknown primary to the neck: II. A review of therapeutic options. Head Neck 2013; 35:286-293.

14. Hsing C-Y, Liu S-A, Wang C-C. Management of unknown primary head and neck squamous cell carcinoma. Am J Otolaryngol 2012; 33:637-638.

15. Villeneuve H, Després P, Fortin B, et al. Cervical lymph node metastases from unknown primary cancer: a single-institution experience with intensitymodulated radiotherapy. Int J Radiat Oncol Biol Phys 2012;82:1866-1871. 